\title{
Understanding off-target effects through hybridization kinetics and thermodynamics
}

\author{
Nafisa N. Nazipova • Svetlana A. Shabalina
}

Received: 16 October 2019 / Accepted: 28 November 2019 / Published online: 10 December 2019

(C) Springer Nature B.V. 2019

In modern biotechnological and medical research, RNA-guided nucleases (RGNs) continue to be highly effective in targeted modification of genomes and the manipulation of gene expression (Sander and Joung 2014; Wang and Wang 2017). In RNA interference (RNAi) and CRISPR (clustered regularly interspaced short palindromic repeats) -Cas (CRISPR-associated protein) systems, RGNs regulate or modify genes through sequence-specific base-pairing between a short interference or single guide RNAs (siRNAs or sgRNAs) and DNA/RNA targets (Bisaria et al. 2017; Shabalina and Koonin 2008). The patterns of base-pairing interactions may modulate RGN binding affinity and reduce off-targeting (Bisaria et al. 2017; Shabalina et al. 2006). RGNs exhibit off-target behavior when interactions and modifications are made not only in the intended location (on-target) but also elsewhere in the genome where sequences are similar to the intended target (off-target) (Klein et al. 2018; Kempton and Qi, 2019). Nucleotide sequence preferences that improve sgRNA efficiency are substantially different for variable CRISPR-based systems (Kim et al., 2019; Slaymaker et al. 2016; Xu

\footnotetext{
N. N. Nazipova $(\bowtie)$

Institute of Mathematical Problems of Biology, Keldysh Institute of Applied Mathematics of Russian Academy of Sciences,

Pushchino, Moscow Region, Russia

e-mail: nafisa.nazipova@gmail.com

\section{S. A. Shabalina $(\bowtie)$}

National Center for Biotechnology Information, National Library of Medicine, National Institutes of Health, Bethesda, MD, USA e-mail: shabalin@ncbi.nlm.nih.gov
}

et al. 2015), which is adapted from diverse bacterial defense systems (Koonin et al. 2017; Makarova et al. 2006). Thus, due in part to growing interest in CRISPRCas variants, this editorial primarily focuses on offtargeting in CRISPR-Cas systems and comparison with RNAi.

CRISPR-Cas proteins are non-specific endonucleases that bind a protospacer adjacent motif (PAM) located in the proximity of the genomic target (Bollen et al. 2018). sgRNA enables the recognition of the target region of interest through complementary base pairing and directs the Cas nuclease there for specific editing. The sgRNA contains a "seed" region, which is especially responsive to mismatches in duplexes with PAMproximal nucleotides, but variants or mutations of the target distal to the PAM also modulate off rates (Boyle et al. 2017). The off-target behavior is not surprising due to the divergence of sgRNA targeting systems where different selective pressures result in optimizations of specificities and other important features, such as turnover rates (Kim et al. 2019).

Preliminary screening of potential candidates and prediction of off-target activities were conducted using not only computational and theoretical approaches but also experimental off-target validation (Zhang et al. 2019; Wienert et al. 2019). Improvement in specificity, on-target cleavage activity, and reduction of off-targetcleavage can be achieved through changes in CRISPRderived nuclease, engineering of sgRNA, and/or CassgRNA delivery modifications. Driving improvements to these parameters is a crucial enabler for RGN-based technologies and the realization of its currently 
untapped potential (Kim et al. 2019; Bisaria et al. 2017). We will also discuss the importance of thermodynamic and kinetic properties for RGN specificity estimation and reducing off-targeting through optimization of sgRNAs. In addition, we briefly touch on the role of enzyme engineering.

\section{Selection of efficient target sites}

The combination of several features defines RGN offtarget effects, including (i) nuclease concentration and features and (ii) target site accessibility, functionality, and uniqueness. The occurrence of complementary sites within the genome, which form highly stable duplexes to guide oligonucleotide, is one of the important determinants of off-target activity. Cas9 and Cas12a (Cpfl) activity can be modulated by chromatin states to varying degrees. Off-targeting is expected to be context dependent because chromatin states and DNA accessibility is tissue/cell- or condition-dependent (Kim et al. 2019). Using the combination of statistical thermodynamics and kinetics, Farasat and Salis (2016) demonstrated that the supercoiling of DNA is an important mechanism tied to the control of Cas9 binding. DNA stretching and bubbles with up to ten mismatches can induce Cas9 off-targeting (Newton et al. 2019). Knockout rates significantly improve when unique target sites are in constitutive upstream exons or in conserved domains/sites with critical gene function. Most of these features were used for computational and theoretical predictions of on-target and off-target activity using different algorithms and web design tools (Zhang et al. 2019; Alkan et al. 2018). Among these methods, data-driven machine learning approaches are very successful at learning rules to model specific datasets, but the models may not be generalizable to new data and systems, or representative of physical or mechanistic relationships (Zhang et al. 2019). Recently, several experimental off-target validation techniques demonstrated successful results, including detection of off-target cleavages throughout the entire genome using approaches, which leveraged advances in next generation sequencing (Wienert et al. 2019).

Empirically derived sets of rules, based primarily on experimental off-targeting data, often include RGN decreased binding affinity to its target (Bisaria et al. 2017), which frequently reduces on-target cleavage. Successful oligonucleotide (sgRNA/siRNA/DNA) targeting is accompanied by an increase in specificity of oligonucleotide, which is defined as the ratio of on-target cleavages to off-targets. In several hybridization experiments and systems, high hybridization specificity and high offtargeting signals (cross-hybridization) are not mutually exclusive (Matveeva et al. 2016, 2018). Notable examples are (i) a model for estimating binding energy of the Cas9-gRNA-DNA complex, based on energy parameters experimentally obtained for relevant interactions between nucleic acids (Alkan et al. 2018), and (ii) a physical framework with rigorous free energy analysis (Zhang et al. 2019) for R-loop formation and sgRNA folding. These models can provide an accurate specificity/efficacy and off-targeting evaluations for sgRNA selections and bridge the gap between experimental structural studies and theoretical predictions. The introduction of nucleic acid duplex energy parameters from experimental measurements (Turner and Mathews 2010) as key components of biophysical models helps to discriminate between highly specific oligonucleotides and improves off-target predictions in different RGN systems, including RNAi (Alkan et al. 2017; Matveeva et al. 2012, 2010, 2007; Shabalina et al. 2006) and CRISPR-Cas systems (Alkan et al. 2018; Farasat and Salis, 2016).

\section{Determinants and kinetic basis for off-target activities}

Despite certain differences in determinants of RNAi and CRISPR-Cas systems, there are important commonalities that could be explained using thermodynamics and hybridization kinetics. In the RNAi studies of mouse Argonaute2 (AGO2) RISC complex formation, several key measurements including association kinetics, equilibrium binding energies, and single turnover cleavage rates allows revealing of important rules for binding and cleavage of targets. Becker and co-authors (Becker et al. 2019) suggested a novel strategy for efficient siRNA design after identifying a specific pattern for guide-target mismatches, which increases the target cleavage rate. These data agree with computational estimations and theoretical predictions of optimal siRNA candidates, where positiondependent patterns of mismatches and thermodynamic features of efficient siRNA candidates exhibited a crucial role (Shabalina et al. 2006). The 
same strategy using an in vitro high-throughput assay was previously applied to evaluate Cas9 binding effectiveness with target sequences with mutations among nucleotides that bind the sgRNA and PAM (Boyle et al. 2017). Mismatch configurations of specific guide positions led to complex nuclease-dCas9 (dead Cas9) dissociation patterns. Significant variation in association and dissociation was noted and attributed to multiple mismatches between sgRNA and DNA at non-seed bases, implying that Cas 9 performance could be influenced by kinetic and thermodynamic adjustment (Boyle et al. 2017).

A comparison of Cas9- and Cas12a-binding experiments showed variable binding kinetics responses to target sequence mutations, which explained why Ca$\mathrm{s} 12 \mathrm{a}$ enables the selection of DNA sequences more precisely than Cas9 (Strohkendl et al. 2018; Boyle et al. 2017). DNA cleavage of both matched and mismatched targets by CRISPR-Cas12a depends on the rate of DNA target binding. Cas12a tightly binds DNA in two distinct kinetic stages, whereas PAM recognition is followed by a rate-limiting R-loop (a hybrid structure of Cas-RNA and target DNA) propagation. The target DNA of Cas12a extend beyond a seed region and has a specific distinguishing pattern of mismatches across much of the R-loop. These observations support the in vivo DNA cleavage patterns and suggest a late transition state for R-loop formation and readily reversible $\mathrm{R}$-loop propagation. Thus, levels of target specificity as well as off-target effects have significant dynamic range across disparate types of nucleases despite the similarity in formation of R-loops served as sequence-specific binding source. Highly efficient nucleases are likely to show more severe off-target activity (Kim et al. 2019).

For Cas nucleases, targeting rules were empirically established by different groups (Klein et al. 2018): (a) the PAM proximal seed region is extremely sensitive to interference from even singlenucleotide mismatches, while significantly diminished sensitivity to mismatches is characteristic of the distal region (individual Cas pattern); (b) offtargets (outside the seed region) are targeted most strongly when mismatches are dispersed; (c) binding exhibited less sensitivity to mismatches than cleavage; and (d) while still maintaining efficiency, target selectivity can be improved by weakened protein DNA interactions (Klein et al. 2018). These rules have already resulted in improvements in the design and prediction strategies of efficient and specific targets (Kim et al. 2019).

\section{Hybridization kinetics of intermolecular interactions and off-target activity}

Several models and approaches based upon thermodynamics and kinetics have the potential to explain off-targeting patterns for CRISPR-Cas and AGO2, as well as for other systems. To go beyond binding energetics, Klein and co-authors (Klein et al. 2018) kinetically modeled formation of guide-target hybrid using characteristics of transition barriers between metastable states of the hybrid with the nuclease. The study demonstrated that mismatch-pattern dependence and seed region can be attributed to the hybridization kinetics, and that the off-targeting rules (a)-(d) (see above) have a kinetic basis. The model showed that kinetically stalled hybridization produced more promiscuous binding than cleavage. The approach also fared favorably when compared with data from different CRISPR-Cas systems, as well as AGO2, and may be applied to any RGN with significant complementarity between guide and target. The study demonstrated that the specificity of engineered systems can be improved without on-target efficiency reduction.

Bisaria and co-authors (Bisaria et al. 2017) analyzed RGN specificity and off-targeting for RNAi and CRISPR-based genome editing. They considered two kinetic regimes: "rapid equilibrium" and "sticky". Dissociation of RGN from the target was faster than cleavage in the first regime and was slow in the second. Several studies discussed approaches for shifting between kinetic regimes to the "rapid-equilibrium" state of RNA targeting and presented evidence that RGNs occur in a "sticky" state (Bisaria et al. 2017) that may be valuable for in vivo RNAi and CRISPR systems (Wang et al. 2006). The described kinetic models explain some details of RGN targeting mechanisms and highlight the fundamental similarity between different RGN systems. Another approach for improving of the CRISPR system specificity is related to engineering of RNA hairpin folding onto sgRNA spacer regions (hp-sgRNAs). Spacer secondary structures affect the characteristics of 
kinetic models including the formation of R-loop and can significantly improve specificity. RNA folding emerged as a key parameter for regulating the activity of CRISPR systems, when applied to five distinct variants of Cas9 and Cas12a (Kocak et al. 2019).

\section{Perspectives}

Attenuating DNA cleavage kinetics can be successfully applied for enhancing gene editing specificity and reducing off-targeting not only to CRISPR systems but also to different engineered nucleases (Becker et al. 2019; Miller et al. 2019). Further understanding of off-targeting mechanisms and basic kinetic features is important for future utility of RGNs, and specifically, in CRISPR-Cas technologies with engineered enzymes as precise genome editing tools. One important future direction is the optimization of multiplexed genome engineering approaches with the possibility of simultaneous modification of multiple genetic elements, which are specifically located in non-coding genome regions (Campa et al. 2019; Reis et al. 2019). Another crucial future prospect is the creation of a platform to characterize kinetic and thermodynamic properties of the growing variety of CRISPR nucleases. Comparison of CRISPR, RNAi, and other RGN systems and analysis of their universal features and descriptive parameters can generate mutually beneficial knowledge and cross-talk between these systems.

Open Access This article is licensed under a Creative Commons Attribution 4.0 International License, which permits use, sharing, adaptation, distribution and reproduction in any medium or format, as long as you give appropriate credit to the original author(s) and the source, provide a link to the Creative Commons licence, and indicate if changes were made. The images or other third party material in this article are included in the article's Creative Commons licence, unless indicated otherwise in a credit line to the material. If material is not included in the article's Creative Commons licence and your intended use is not permitted by statutory regulation or exceeds the permitted use, you will need to obtain permission directly from the copyright holder. To view a copy of this licence, visit http://creativecommons.org/licenses/by/4.0/.

\section{References}

Alkan F, Wenzel A, Anthon C, Havgaard JH, Gorodkin J. CRISPR-Cas9 off-targeting assessment with nucleic acid duplex energy parameters. Genome Biol. 2018;19:177. https://doi.org/10.1186/s13059-018-1534-x.

Alkan F, Wenzel A, Palasca O, Kerpedjiev P, Rudebeck AF, Stadler PF, et al. RIsearch2: suffix array-based large-scale prediction of RNA-RNA interactions and siRNA off-targets. Nucleic Acids Res. 2017;45:e60. https://doi.org/10.1093 /nar/gkw1325.

Becker WR, Ober-Reynolds B, Jouravleva K, Jolly SM, Zamore PD, Greenleaf WJ. High-throughput analysis reveals rules for target RNA binding and cleavage by AGO2. Mol Cell. 2019;75:741-55.e11.

Bisaria N, Jarmoskaite I, Herschlag D. Lessons from enzyme kinetics reveal specificity principles for RNA-guided nucleases in RNA interference and CRISPR-based genome editing. Cell Syst. 2017;4:21-9. https://doi.org/10.1016/j. cels.2016.12.010.

Bollen Y, Post J, Koo BK, Snippert HJG. How to create state-ofthe-art genetic model systems: strategies for optimal CRISPR-mediated genome editing. Nucleic Acids Res. 2018;46:6435-54.

Boyle EA, Andreasson JOL, Chircus LM, Sternberg SH, Wu MJ, Guegler CK, et al. High-throughput biochemical profiling reveals sequence determinants of dCas9 off-target binding and unbinding. Proc Natl Acad Sci U S A. 2017;114:5461-6.

Campa CC, Weisbach NR, Santinha AJ, Incarnato D, Platt RJ. Multiplexed genome engineering by Cas12a and CRISPR arrays encoded on single transcripts. Nat Methods. 2019;16: 887-93.

Farasat I, Salis HM. A biophysical model of CRISPR/Cas9 activity for rational design of genome editing and gene regulation. PLoS Comput Biol. 2016;12:e1004724. https://doi. org/10.1371/journal.pcbi.1004724.

Kempton HR, Qi LS. When genome editing goes off-target. Science. 2019;364:234-6.

Kim D, Luk K, Wolfe SA, Kim JS. Evaluating and enhancing target specificity of gene-editing nucleases and deaminases. Annu Rev Biochem. 2019;88:191-220. https://doi. org/10.1146/annurev-biochem-013118-111730.

Klein M, Eslami-Mossallam B, Arroyo DG, Depken M. Hybridization kinetics explains CRISPR-Cas off-targeting rules. Cell Rep. 2018;22:1413-23.

Kocak DD, Josephs EA, Bhandarkar V, Adkar SS, Kwon JB, Gersbach CA. Increasing the specificity of CRISPR systems with engineered RNA secondary structures. Nat Biotechnol. 2019;37:657-66.

Koonin EV, Makarova KS, Zhang F. Diversity, classification and evolution of CRISPR-Cas systems. Curr Opin Microbiol. 2017;37:67-78.

Makarova KS, Grishin NV, Shabalina SA, Wolf YI, Koonin EV. A putative RNA-interference-based immune system in prokaryotes: computational analysis of the predicted enzymatic machinery, functional analogies with eukaryotic RNAi, and hypothetical mechanisms of action. Biol Direct. 2006;1:7. https://doi.org/10.1186/1745-6150-1-7.

Matveeva O, Nechipurenko Y, Rossi L, Moore B, Saetrom P, Ogurtsov AY, et al. Comparison of approaches for rational siRNA design leading to a new efficient and transparent method. Nucleic Acids Res. 2007;35:e63. https://doi. org/10.1093/nar/gkm088.

Matveeva OV, Kang Y, Spiridonov AN, Saetrom P, Nemtsov VA, Ogurtsov AY, et al. Optimization of duplex stability and 
terminal asymmetry for shRNA design. PLoS One. 2010;5: e10180. https://doi.org/10.1371/journal.pone.0010180.

Matveeva OV, Nazipova NN, Ogurtsov AY, Shabalina SA. Optimized models for design of efficient miR30-based shRNAs. Front Genet. 2012;3:163. https://doi.org/10.3389 /fgene.2012.00163.

Matveeva OV, Nechipurenko YD, Riabenko E, Ragan C, Nazipova NN, Ogurtsov AY, et al. Optimization of signalto-noise ratio for efficient microarray probe design. Bioinformatics. 2016;32:i552-8. https://doi.org/10.1093 /bioinformatics/btw451.

Matveeva OV, Ogurtsov AY, Nazipova NN, Shabalina SA. Sequence characteristics define trade-offs between on-target and genome-wide off-target hybridization of oligoprobes. PLoS One. 2018;13:e0199162. https://doi.org/10.1371 /journal.pone.0199162.

Miller JC, Patil DP, Xia DF, Paine CB, Fauser F, Richards HW, et al. Enhancing gene editing specificity by attenuating DNA cleavage kinetics. Nat Biotechnol. 2019;37:945-52. https://doi.org/10.1038/s41587-019-0186-z.

Newton MD, Taylor BJ, Driessen RPC, Roos L, Cvetesic N, Allyjaun S, et al. DNA stretching induces Cas9 off-target activity. Nat Struct Mol Biol. 2019;26:185-92.

Reis AC, Halper SM, Vezeau GE, Cetnar DP, Hossain A, Clauer PR, et al. Simultaneous repression of multiple bacterial genes using nonrepetitive extra-long sgRNA arrays. Nat Biotechnol. 2019;37:1294-301. https://doi.org/10.1038 /s41587-019-0286-9.

Sander JD, Joung JK. CRISPR-Cas systems for editing, regulating and targeting genomes. Nat Biotechnol. 2014;32:347-55.

Shabalina SA, Spiridonov AN, Ogurtsov AY. Computational models with thermodynamic and composition features improve siRNA design. BMC Bioinformatics. 2006;7:65. https://doi.org/10.1186/1471-2105-7-65.

Shabalina SA, Koonin EV. Origins and evolution of eukaryotic RNA interference. Trends Ecol Evol. 2008;23:578-87.
Slaymaker IM, Gao L, Zetsche B, Scott DA, Yan WX, Zhang F. Rationally engineered Cas9 nucleases with improved specificity. Science. 2016;351:84-8.

Strohkendl I, Saifuddin FA, Rybarski JR, Finkelstein IJ, Russell R. Kinetic basis for DNA target specificity of CRISPR-Cas12a. Mol Cell. 2018;71:816-24.e3.

Turner DH, Mathews DH. NNDB: the nearest neighbor parameter database for predicting stability of nucleic acid secondary structure. Nucleic Acids Res. 2010;38(Database issue): D280-2. https://doi.org/10.1093/nar/gkp892.

Wang X-H, Aliyari R, Li W-X, Li H-W, Kim K, Carthew R, et al. RNA interference directs innate immunity against viruses in adult drosophila. Science. 2006;312:452-4. https://doi. org/10.1126/science.1125694.

Wang W, Wang X. Single-cell CRISPR screening in drug resistance. Cell Biol Toxicol. 2017;33(3):207-10. https://doi. org/10.1007/s10565-017-9396-7.

Wienert B, Wyman SK, Richardson CD, Yeh CD, Akcakaya P, Porritt MJ, et al. Unbiased detection of CRISPR off-targets in vivo using DISCOVER-Seq. Science. 2019;364:286-9. https://doi.org/10.1126/science.aav9023.

Xu H, Xiao T, Chen CH, Li W, Meyer CA, Wu Q, et al. Sequence determinants of improved CRISPR sgRNA design. Genome Res. 2015;25:1147-57. https://doi.org/10.1101 /gr.191452.115.

Zhang D, Hurst T, Duan D, Chen SJ. Unified energetics analysis unravels SpCas9 cleavage activity for optimal gRNA design. Proc Natl Acad Sci U S A. 2019;116:8693-8. https://doi. org/10.1073/pnas.1820523116.

Publisher's note Springer Nature remains neutral with regard to jurisdictional claims in published maps and institutional affiliations. 